Ann. Zootech., I970, 19 (4), 465-469.

NOTE

\title{
MENSURATIONS, IN VIVO ET POSTMORTEM, ET CROISSANGE DE L'INTESTIN GRELE CHEZ LE PORC
}

\author{
J.-P. LAPLACE \\ avec la collaboration technique de C. Germain \\ Station de Recherches sur l'Élevage des Porcs, \\ Centre national de Recherches zootechniques, \\ 78 - Jouy-en-Josas \\ Institut national de la Recherche agronomique
}

\section{RÉSUMÉ}

La croissance en longueur et en poids de l'intestin grêle a été étudiée sur 47 porcs de race Large White de poids variant entre $\mathrm{I} 2$ et $\mathrm{I} 6 \mathrm{I} \mathrm{kg}$. Les équations de régression font apparaître la prédominance de la croissance pondérale sur la croissance en longueur de l'intestin, au-delà de $30 \mathrm{~kg}$ de poids vif. Les mesures de longueur intestinale effectuées in vivo, ou après abattage par anesthésie électrique, ou encore après sacrifice par anesthésie endoveineuse sont comparées. Une contraction de 9 à I 5 p. Ioo est observée selon le mode d'abattage.

\section{INTRODUC'TION}

Au terme d'expériences de nutrition, les mensurations digestives constituent souvent un élément important de connaissance des effets liés au régime, ou d'explication des résultats expérimentaux. On connaît en effet l'intensité des modifications morphologiques et fonctionnelles, consécutives à l'ingestion de régimes contenant un diluant inerte, ou à une restriction ali mentaire (Dowling, 1967). Le problème se pose tout particulièrement pour le porc, lors d'incorporation de cellulose dans la ration (HEnRy et Étienne, I969), ou lors d'étude de l'influence du niveau d'alimentation et de restrictions alimentaires vis-à-vis de la composition corporelle (DEsmouLIN, I969). La complexité, pour une expérimentation sur des lots i mportants d'animaux, de la technique de mesure proposée par Vodovar et al., (1964), et la variabilité aléatoire des mensurations d'abattoir, font que ce contrôle doit généralement être abandonné. Notre but est ici de 
comparer aux résultats de Vodovar, ceux obtenus par une méthode plus simple récemment proposée (LAPLACE, I970), et de chiffrer l'écart entre cette mensuration post-mortem et la mesure in vivo telle qu'elle peut être pratiquée au cours d'une intervention chirurgicale.

\section{MATÉRIEL ET MÉTHODES}

Soixante porcs de race Large-White ont été utilisés dans deux types d'étude :

D'une part la totalité de la masse viscérale de 47 animaux d'un poids variant entre 12 et I6r $\mathrm{kg}$, a été prélevée après anesthésie électrique et saignée. L'estomac et l'intestin grêle sont libérés du mésentère par section ménageant la séreuse intestinale. La mesure de la longueur de l'intestin est effectuée par étalement sur une surface graduée. Puis après lavage, essorage et expulsion du mucus, l'estomac et l'intestin grêle sont pesés séparément. Parallèlement ont été pesés le foie et les reins.

D'autre part, 13 porcs de $70 \mathrm{~kg}$ environ, ont été soumis à une mensuration intestinale in vivo. Celle-ci est effectuée dans les conditions opératoires antérieurement décrites (LAPLACE, 1970). La mesure elle-même est réalisée en appliquant sur la paroi intestinale, à égale distance des bords mésentérique et anti-mésentérique, une cordelette de nylon d'une longueur déterminée. Des points de soie $n^{\circ}$ o sont mis en place aux intervalles choisis. Chez ro de ces porcs, un seul tronçon de $4 \mathrm{~m}$ a été ainsi délimité. Huit jours après l'opération, ces animaux sont abattus par anesthésie électrique et saignée. Pour les trois autres porcs, la totalité de l'intestin grêle a été marquée de 2 en $2 \mathrm{~m}$ ou de 4 en $4 \mathrm{~m}$. Deux d'entre eux ont été sacrifiés ( $8 \mathrm{e}$ jour), par surcharge anesthésique de Nembutal (N.D.) par voie endoveineuse, suivie de saignée, l'un d'eux ayant été préalablement soumis à une sévère restriction alimentaire (zoo g par jour d'aliment "croissance " pendant 5 jours). Le troisième porc a subi l'anesthésie électrique et la saignée classique.

\section{RÉSULTATS E'T DISCUSSION}

\section{a) Croissance viscérale entre 12 et $161 \mathrm{~kg}$}

Le calcul, sur l'ensemble des 47 porcs, des équations de régression des diverses mesures par rapport au poids vif, et des coefficients de corrélation fait apparaître une liaison importante pour trois des données :

$$
\begin{array}{lcc}
\text { Poids du foie ..... } y=\mathrm{II}, 6 x+477,7 & r=0,87 \\
\text { Poids des reins.... } & y=2,0 x+78,6 & r=0,89 \\
\text { Poids de l'estomac } & y=4, \mathrm{I} x+\mathbf{I} 40,5 & r=0,9 \mathrm{I}
\end{array}
$$

La liaison entre le poids vif et la longueur de l'intestin grêle est moindre, considérée sur l'ensemble de la population $\left(y=2,5 x+\mathrm{I}_{4} \mathrm{II}, 9 ; r=0,5 \mathrm{I}\right)$. La subdivision des animaux en deux classes fournit pour la période 12 à $30 \mathrm{~kg}$ une liaison étroite $(y=23,5 x+892,5$; $r=0,93)$; alors qu'entre 30 et I6I kg $(y=\mathrm{I}, 3 x+\mathrm{I} .438,9)$ la dispersion des mesures ne permet qu'un coefficient $r$ de 0,2 .

Un phénomène analogue survient dans le cas du poids de l'intestin grêle :

$$
\begin{array}{lll}
\text { Ensemble de la population }: y=7,5 x+677,2 & r=0,76 \\
\text { Sous-population (12-30 kg) }: y=44,6 x-14 \mathrm{I}, 7 & r=0,97 \\
\text { Sous-population (30-16I kg) :y=5,2x+915,I } & r=0,47
\end{array}
$$




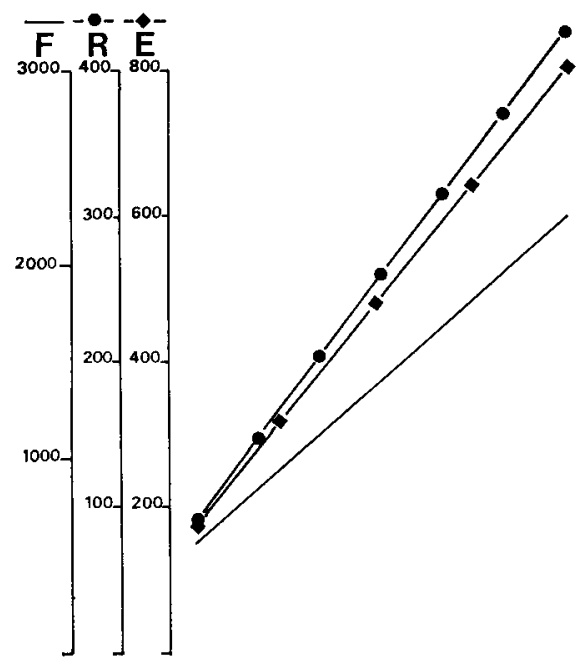

020

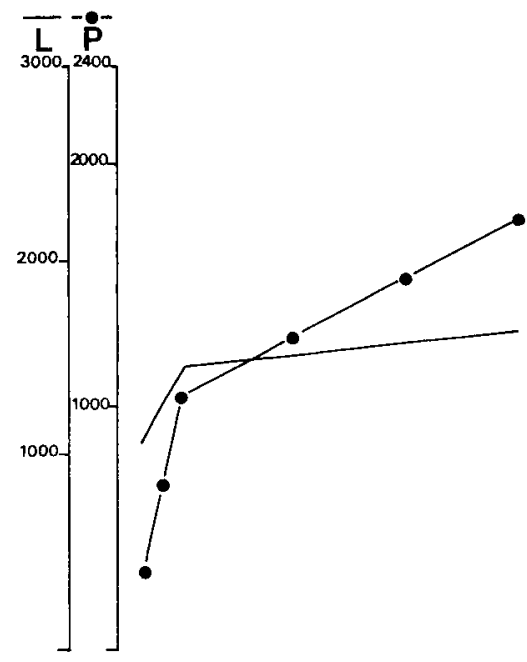

$0 \quad 20$

FIG. I. - Droites descriptives des équations de régression en fonction du poids vif de l'animal du poids du foie (F), des reins ( $\mathrm{R})$, de l'estomac $(\mathrm{E})$, de la longueur $(\mathrm{L})$, et $d u$ poids (P) de l'intestin grêle

(poids en $\mathrm{g}$; longueur en $\mathrm{cm}$ )

En définitive, l'accroissement pondéral du foie, des reins, et même de l'estomac, est en relation directe avec l'évolution du poids vif de l'animal au cours d'une tranche de vie importante (I2 à $16 \mathrm{r} \mathrm{kg}$ ). Par contre, les mensurations de l'intestin grêle évoluent différemment dans les deux sous-populations choisies. La longueur totale de celui-ci croit d'environ $4 \mathrm{~m}$ (de ro à I $4 \mathrm{~m}$ ) entre 12 et $30 \mathrm{~kg}$, et de $3 \mathrm{~m}$ seulement (de 14 à $17 \mathrm{~m}$ ) entre 30 et $\mathrm{I} 6 \mathrm{I} \mathrm{kg}$ ). Le faible degré de corrélation au-delà de $30 \mathrm{~kg}$, rend compte de la dispersion croissante des mesures, liée à l'importance des effets de l'inégalité des niveaux d'ingestion. L'incidence de cette variation est moindre en ce qui concerne le poids de l'intestin grêle, mais là aussi, les 2 équations obtenues décrivent correctement les observations.

\section{b) Comparaison des mensurations in vivo et post-mortem}

Les mesures de longueur rapportées sont, en valeur absolue, plus faibles que celles de VodoVAR et al., (I964), supposées identiques aux mesures réelles in vivo. L'essentiel de cette différence apparaît comme la conséquence d'une rétraction intestinale lors du sacrifice. En effet, l'examen. du tableau $\mathrm{I}$ montre que la réduction de la valeur absolue de la mesure après abattage classique atteint I2 à I 5 p. Ioo, selon que le calcul est effectué sur Io segments de $4 \mathrm{~m}$ appartenant à ro animaux ou sur 5 segments de $2 \mathrm{~m}$ appartenant à un même animal. La réduction n'est que de 9 p. roo après sacrifice au Nembutal, ce qui souligne l'effet de contraction lié à l'électrocution. Cet effet peut du reste atteindre exceptionnellement $20 \mathrm{p}$. Ioo chez certains animaux à forte excitabilité neuro-musculaire. Enfin, le porc $\mathrm{C}$, soumis à une sévère restriction alimentaire présente, en plus de la réduction de $9 \mathrm{p}$. Ioo inhérente au sacrifice, une réduction de longueur des segments d'intestin grêle de l'ordre de $\mathbf{} 6$ p. Ioo.

En conclusion, il apparaît que la technique simple de mensuration intestinale post-mortem proposée permet de définir de façon satisfaisante la longueur réelle de l'intestin chez l'animal vivant, sous réserve de relever les mesures de I 2 à I 5 p. roo lors d'anes thésie électrique classique, 


\section{TABI,EAU I}

Mesures post-mortem, selon le mode d'abattage, de segments d'intestin préalablement marqués in vivo. Le porc $C$ a été soumis à une sévère restriction alimentaire au cours des 5 jours précédant l'abattage.

\begin{tabular}{|c|c|c|c|c|c|}
\hline \multirow[b]{4}{*}{ Mesure per-opératoire } & \multicolumn{5}{|c|}{ Mode d'abattage } \\
\hline & \multicolumn{2}{|c|}{ Anesthésie électrique } & \multicolumn{3}{|c|}{ Nemtubal (N.D.) } \\
\hline & 10 pores & pore $A$ & porc B & \multicolumn{2}{|c|}{ porc $\mathrm{C}$} \\
\hline & $400 \mathrm{~cm}$ & $200 \mathrm{~cm}$ & $200 \mathrm{~cm}$ & $200 \mathrm{~cm}$ & $400 \mathrm{~cm}$ \\
\hline Mesure post-mortcm & $\begin{array}{l}258 \\
383 \\
362 \\
363 \\
362 \\
327 \\
368 \\
391 \\
38^{\prime} \\
327\end{array}$ & $\begin{array}{l}168 \\
170 \\
176 \\
182 \\
160\end{array}$ & $\begin{array}{l}193 \\
18 \mathbf{t} \\
172 \\
186 \\
185 \\
176\end{array}$ & $\begin{array}{l}122 \\
165 \\
150\end{array}$ & $\begin{array}{l}295 \\
315\end{array}$ \\
\hline $\bar{x} \pm s$ & $\begin{array}{r}352,50 \\
+\quad 12,51\end{array}$ & $\begin{array}{r}171,20 \\
+\quad 3,72\end{array}$ & $\begin{array}{r}182,66 \\
\pm \quad 3,07\end{array}$ & 145,66 & 305,00 \\
\hline Réduction en p. 100 & 12 & 15 & 9 & 27 & 24 \\
\hline
\end{tabular}

ou de 9 à Io p. ıoo lors de sacrifice par injection endoveineuse d'anesthésique. Par rapport à la croissance de l'animal, la croissance pondérale de l'intestin grêle est plus importante que la croissance en longueur au-delà de $30 \mathrm{~kg}$ de poids vif. L'effet de la restriction alimentaire sur la longueur digestive semble important chez le porc, et mérite un examen approfondi.

Reçu pour publication en novembre 1970.

\section{SUMMARY}

IN VIVO AND POST MORTEM MEASURES OF THE SMALL INTESTINE GROWTH IN THE PIG

Growth in weight and lenght of the small intestine was studied in 47 Large White pigs between I2 and $16 \mathrm{r} \mathrm{kg}$ live weight. The calculation of regression coefficients shows that after $30 \mathrm{~kg}$ live weight, the increase in weight is higher than the increase in lenght. A comparison was made between the measures of the intestinal lenght carried out in vivo, after slaughter by electric anaesthesia or by endoveineous anaesthesia. A 9 to $\mathrm{r} 5 \mathrm{p}$. roo contraction was observed according to the mode of slaughtering. 


\section{RÉFÉRENCES BIBLIOGRAPHIQUES}

Desmoulix B., I969. Influence des méthodes de présentation du repas sur les performances du porc soumis à un plan de rationnement. Journ. Recherche Porcine en France, I. N. R. A., Paris, I Vol., 73-76.

Dowling R. H., 1967. Compensatory changes in intestinal absorption. Br. Med. Bull., 23, $275-278$. Henry H., Étienne M., Ig69. Effets nutritionnels de l'incorporation de cellulose purifiée dans le régime du porc en croissance-finition. Io Influence sur l'utilisation digestive des nutriments. Ann. Zootech., 18, 337-357.

LAPlace J.-P., x970. Résections intestinales chez le porc. I. Observations préliminaires. Ann.Zootech., $19,287-302$.

Vodovar N., Flanzy J., Françors A.-C., r964. Intestin grêle du porc. I. Dimensions en fonction de l'âge et du poids ; étude de la jonction du canal cholédoque et du canal pancréatique à celui-ci. Ann. Biol. anim. Bioch. Biophys., 4, 27-34. 